\title{
Lévy stable distributions for velocity and velocity difference in systems of vortex elements
}

\author{
I. A. Min ${ }^{\mathrm{a})}$ \\ Graduate Aeronautical Laboratories, California Institute of Technology, Pasadena, California 91125 \\ I. Mezić \\ Department of Mechanical and Environmental Engineering, University of California, \\ Santa Barbara, California 93106-5070
}

A. Leonard

Graduate Aeronautical Laboratories, California Institute of Technology, Pasadena, California 91125

(Received 11 October 1995; accepted 11 January 1996)

\begin{abstract}
The probability density functions (PDFs) of the velocity and the velocity difference field induced by a distribution of a large number of discrete vortex elements are investigated numerically and analytically. Tails of PDFs of the velocity and velocity difference induced by a single vortex element are found. Treating velocities induced by different vortex elements as independent random variables, PDFs of the velocity and velocity difference induced by all vortex elements are found using limit distribution theorems for stable distributions. Our results generalize and extend the analysis by Takayasu [Prog. Theor. Phys. 72, 471 (1984)]. In particular, we are able to treat general distributions of vorticity, and obtain results for velocity differences and velocity derivatives of arbitrary order. The PDF for velocity differences of a system of singular vortex elements is shown to be Cauchy in the case of small separation $r$, both in 2 and 3 dimensions. A similar type of analysis is also applied to non-singular vortex blobs. We perform numerical simulations of the system of vortex elements in two dimensions, and find that the results compare favorably with the theory based on the independence assumption. These results are related to the experimental and numerical measurements of velocity and velocity difference statistics in the literature. In particular, the appearance of the Cauchy distribution for the velocity difference can be used to explain the experimental observations of Tong and Goldburg [Phys. Lett. A 127, 147 (1988); Phys. Rev. A 37, 2125, (1988); Phys. Fluids 31, 2841 (1988)] for turbulent flows. In addition, for intermediate values of the separation distance, near exponential tails are found. (C) 1996 American Institute of Physics. [S1070-6631(96)01605-1]
\end{abstract}

\section{INTRODUCTION}

We study the probability density function (PDF) of velocity and velocity difference associated with a discrete, deterministic vortex system. There are at least two reasons for considering such a system: firstly, the discrete vortex model as a computational technique has seen much development in recent years, ${ }^{1,2}$ and has proven to be a useful tool for flow computation. Secondly, recent visualizations of fine-scales of turbulence (experimental and numerical) have shown the presence of distinct vortex elements as being the key driver of the flow. ${ }^{3,4}$ These facts are clearly not unrelated. Further, Saffman ${ }^{5}$ proposes that " . ...turbulence should be modeled or described as the creation, evolution, interaction and decay of these [discrete vortical] structures....' In this context, it is of obvious interest to find the statistical behavior of the velocity field associated with a collection of discrete vortex elements, singular or with a core, and compare the results obtained to numerical simulations and experiments. Novikov $^{6}$ has made a step in this direction by finding the energy spectrum of a velocity field induced by a system of $N$ singular vortices in the plane. In this work we study nu-

\footnotetext{
${ }^{a}$ Current address: Fluid Mechanics Department, The Aerospace Corp., P.O. Box 92957, Los Angeles, California 90009. Electronic mail: min@1vtd2.aero.org
}

merically the induced velocity PDF itself, for the system of vortex elements in two dimensions, and PDFs of its derivatives of arbitrary order. We also propose a theory for the observed results based on some assumptions discussed below, and extend this theory to three-dimensional situations. We do this by first analyzing the velocity field induced by a single vortex and then using limit distribution theorems to include the effects of the contribution of all the vortices. Investigation of the statistics of the velocity field induced by the motion of $N$ singular vortices and vortices with a finite core is the main thrust of this paper. A connection with experimental and numerical measurements of velocity and velocity difference distributions in turbulent flows is attempted.

The main assumptions in the analytical part of this work are that the system of vortex elements is ergodic in its phase space (see Section II and the Appendix), and that the velocities induced by different vortices can be treated as independent random variables. For an investigation of the ergodicity assumption for a system of $N$ point vortices, see Ref. 10 . We also assume that the probability density of a vortex element in the physical space is not concentrated on a fractal set, so that it is an ordinary, two or three dimensional, probability distribution. These assumptions are sufficient to produce a velocity difference PDF which can be used to explain experimental results by Tong and Goldburg. Tong and 
Goldburg's ${ }^{7-9}$ experimental measurements of relative velocity in turbulent flows show a PDF that is well approximated by the product of a Lorentzian (Cauchy distribution) and a Gaussian function. We show in this paper the conditions under which these functions are produced for the $N$-vortex model. We also provide an explanation (backed by numerical experiments) for the appearance of near-exponential tails that are often seen in turbulence experiments.

In discussing the limit distributions of sums of the velocity or velocity difference contributions from single vortices, we will be making use of stable distributions (also often referred to as Lévy stable distributions). A brief description of stable distributions can be stated as follows: Consider independent random variables $X_{1}, X_{2}, \ldots$ and the sum $X_{n}=X_{1}+X_{2}+\ldots$, where the variables $X_{1}, X_{2}, \ldots$ have the same distribution $R$. The distribution $R$ is stable if $X_{n}$ has the same distribution as $X_{i}$ (i.e., $R$ ) apart from norming constants. See Feller ${ }^{11}$ for more detailed description and examples. The importance of stable distributions in physical processes has been highlighted by Mandelbrot, ${ }^{12}$ Montroll and Shlesinger ${ }^{13}$ and Takayasu, ${ }^{14}$ among others (see also Shlesinger et al. ${ }^{15}$ for a recent collection of papers).

The history of investigation of systems of point vortices is long. The statistical physics approach has been applied to the problem of a large number of vortices (see Ref. 16, for example). Other authors have used the vortex element approach to solve interesting flow problems (see Ref. 1). The integrability and chaotic dynamics of the few vortex problem (see Refs. 17, 18, and 10) and the references therein) has been studied intensely. Recently, renewed interest in systems of $\mathrm{N}$ interacting vortices has been sparked by the observation that two dimensional random divergence free fields get organized into distinct vortical structures under the dynamics of Navier-Stokes equations (see, e.g., Ref. 19). Of particular interest are chaotic advection and dispersion of fluid particles in a velocity field produced by $N$ point vortices (see Viecelli ${ }^{20}$ and Babiano et al. ${ }^{21}$ ). Our work may shed some light on these issues by providing information about the statistics of the velocity field that produces chaotic motion and dispersion of fluid particles.

An earlier paper by Takayasu ${ }^{14}$ contains some points in common with the analytical part of our work; namely, the idea of decomposition of velocity to contributions from individual vortices, the assumption of independence, and the importance of stable distributions in describing the PDF of the velocity. We introduce several new ideas that build on this work: the investigation of velocity difference PDF (a useful parameter in turbulence, as discussed below), a more general derivation, introduction of non-singular vorticity, and an attempt to relate these results to those of turbulent flows. Takayasu predicts that the PDF of velocity induced by vortex elements in three dimensions is the Holtzmark distribution. However, there is no known experimental or numerical evidence of the Holtzmark distribution for the velocity PDF $p_{u}(u)$ in turbulence. It is often accepted that $p_{u}(u)$ in a turbulent flow is nearly normally distributed; this is easily accounted for with the use of vortex blobs or singular vortex filaments.

In view of the increasing use of vortex methods ${ }^{22,1}$ of flow computation where discrete, Lagrangian vortex elements are used to simulate turbulent flows, we hope to provide an understanding of the range of validity of these models for gathering statistics. In particular, it follows from our work that PDFs of velocity induced by a system of $\mathrm{N}$ vortices with a finite core can have long non-Gaussian tails if the cutoff parameter of the core is small.

In section II we investigate the PDF of the velocity induced by a single vortex element moving under the influence of $N$ vortex elements, under the assumptions described above. The construction of the tails of the PDF for the velocity at a fixed location due to a single vortex, is presented. The PDF associated with the velocity due to all the vortices, follows from the use of limit distribution theorems. The same construction is used to analyze the velocity difference PDF. In section IV a connection is made with some experimental data. In section $\mathrm{V}$, numerical simulations with $N$-vortices in two dimensions are described, and the results are favorably compared with analytical predictions. In Appendix A we show that a sufficient condition for an assumption on reduced probability densities is ergodicity of a system of vortex elements in their phase space.

\section{THE VELOCITY AND VELOCITY DIFFERENCE FIELD DUE TO A SINGLE VORTEX}

The two-dimensional singular vortex case is discussed in detail first; the vortex blob and the three-dimensional cases follow naturally.

\section{A. Two-dimensional vorticity distribution}

The two-dimensional vorticity field represented by a collection of $N$ singular vortices is given by

$$
\omega=\sum_{i=1}^{N} \Gamma_{i} \delta\left(\mathbf{x}-\mathbf{x}_{i}\right),
$$

where $\delta$ denotes the Dirac delta function.

The velocity at point $\mathbf{x}$ induced by the above distribution of vorticity is

$$
\begin{aligned}
& u(\mathbf{x})=-\frac{1}{2 \pi} \sum_{i=1}^{N} \frac{\Gamma_{i}\left(y-y_{i}\right)}{\left|\mathbf{x}-\mathbf{x}_{i}\right|^{2}}=\sum_{i=1}^{N} u_{i}(\mathbf{x}), \\
& v(\mathbf{x})=\frac{1}{2 \pi} \sum_{i=1}^{N} \frac{\Gamma_{i}\left(x-x_{i}\right)}{\left|\mathbf{x}-\mathbf{x}_{i}\right|^{2}}=\sum_{i=1}^{N} v_{i}(\mathbf{x}),
\end{aligned}
$$

where the $\mathbf{x}_{i}$ are the vortex positions and $u_{i}(\mathbf{x})$ is that portion of the $x$ component of velocity induced by the $i$ th vortex:

$$
\begin{aligned}
& u_{i}(\mathbf{x}) \equiv \frac{-\Gamma_{i}}{2 \pi} \frac{\left(y-y_{i}\right)}{\left|\mathbf{x}-\mathbf{x}_{i}\right|^{2}}=-\frac{\Gamma_{i}}{2 \pi} \frac{\sin \theta_{i}}{\rho_{i}}, \\
& \rho_{i} \equiv\left|\mathbf{x}-\mathbf{x}_{i}\right|, \\
& \theta_{i} \equiv \tan ^{-1}\left(\frac{y-y_{i}}{x-x_{i}}\right),
\end{aligned}
$$

and similarly, the $y$ component induced by vortex $i$ is

$$
v_{i}(\mathbf{x}) \equiv \frac{\Gamma_{i}}{2 \pi} \frac{\left(x-x_{i}\right)}{\left|\mathbf{x}-\mathbf{x}_{i}\right|^{2}}=\frac{\Gamma_{i}}{2 \pi} \frac{\cos \theta_{i}}{\rho_{i}} .
$$


For the sake of simplicity of derivation, consider the norm of the velocity, $|\mathbf{u}|$ rather than its components (an analogous derivation in terms of the components is straightforward, but a little more cumbersome). Let us define $p_{u_{i}}\left(\left|\mathbf{u}_{i}\right| ; \mathbf{x} \mid p_{i}\left(\mathbf{x}_{i}\right)\right)$ as the probability density of the norm of the velocity due to a single vortex $i$, measured at $\mathbf{x}$. The reduced PDF $p_{i}\left(\mathbf{x}_{i}\right)$ is defined through the expression $p_{i}\left(\mathbf{x}_{i}\right) d \mathbf{x}_{i}=$ Probability $\left\{\right.$ vortex $\left.i \in d \mathbf{x}_{i}\right\}$ and $\int p_{i}\left(\mathbf{x}_{i}\right) d \mathbf{x}_{i}=1$. $\mathbf{x}_{i}$ is the position of a single vortex $i$ (see Lundgren and Pointin ${ }^{16}$ ).

Let us now restrict the discussion to the case in which all the vortices are of the same sign and magnitude, $\Gamma_{i}$. That $p_{i}\left(\mathbf{x}_{i}\right)$ is independent of vortex label $i$ if ergodicity of the vortex system is assumed, follows from the considerations in the Appendix. From now on we assume ergodicity and denote PDF for the position of a single vortex by $p_{1}\left(\mathbf{x}_{1}\right)$. For notational convenience we will henceforth denote $p_{u_{i}}\left(\left|\mathbf{u}_{i}\right| ; \mathbf{x} \mid p_{1}\left(\mathbf{x}_{1}\right)\right)$ by $p_{u_{i}}\left(\left|\mathbf{u}_{i}\right|\right)$, neglecting the dependence on $\mathbf{x}$. The probability density associated with the velocity due to all $N$ vortices at $\mathbf{x}$ is denoted $p_{u}(|\mathbf{u}|)$, again neglecting the dependence on $\mathbf{x}$.

We are interested only in the high $\left|\mathbf{u}_{i}\right|$ tails of $p_{u_{i}}\left(\left|\mathbf{u}_{i}\right|\right)$. Consider the probability $P\left(\left|\mathbf{u}_{i}\right|>U\right)$ that the norm of the velocity induced by a single vortex is bigger than $U$. This probability is proportional to $I=\int_{B} p_{1}\left(\mathbf{x}_{1}\right) d \mathbf{x}_{1}$, where $B$ is a ball of radius $\rho=\Gamma / 2 \pi U$. If we assume that the PDF for vortex position, $p_{1}\left(\mathbf{x}_{1}\right)$ is well-behaved (not concentrated on some fractal set of dimension less than 2) and strictly positive, then $I \sim \rho^{2}$ when $\rho$ is small ( $U$ large). Thus we obtain

$$
P\left(\left|\mathbf{u}_{i}\right|>U\right) \sim U^{-2},
$$

for large $U$. Assuming the above function has a derivative, that derivative is exactly $p_{u_{i}}\left(\left|\mathbf{u}_{i}\right|\right)$ and

$$
p_{u_{i}}\left(\left|\mathbf{u}_{i}\right|\right) \sim\left|\mathbf{u}_{i}\right|^{-3},
$$

for large $\left|\mathbf{u}_{i}\right|$.

\section{B. Non-singular vortex case (vortex blobs)}

The singular point vortex representation can be smoothed out to get a non-singular vortex blob. There are several schemes to do this. We will work with the simple algebraic core method which has the following vorticity distribution:

$$
\omega(\mathbf{x}, t)=\frac{1}{\pi} \sum_{i=1}^{N} \frac{\Gamma_{i} \delta_{c}^{2}}{\left(\delta_{c}^{2}+\left|\mathbf{x}-\mathbf{x}_{i}\right|^{2}\right)^{2}},
$$

where $\delta_{c}$ is a constant. Extension to other smoothing schemes is straightforward. The norm of the velocity induced at $\mathbf{x}$ by a vortex at a distance $\rho=\left|\mathbf{x}-\mathbf{x}_{i}\right|$ is

$$
\left|\mathbf{u}_{i}(\rho)\right|=-\frac{\Gamma_{i} \rho}{2 \pi\left(\rho^{2}+\delta_{c}^{2}\right)},
$$

and there is clearly an upper bound to the value of $\left|\mathbf{u}_{i}\right|$ given by $\left|\mathbf{u}_{i}\right|_{\max }=\Gamma_{i} /\left(4 \pi \delta_{c}\right)$ at $\rho=\delta_{c}$. So the finite core parameter $\delta_{c}$ acts as a cutoff such that $p_{i}\left(\left|\mathbf{u}_{i}\right|\right)$ is zero for $\left|\mathbf{u}_{i}\right|>\left|\mathbf{u}_{i}\right|_{\max }$. Note that this allows for the existence of a mean as well as a variance for the velocity; in the case of the singular vortex, the variance does not exist.

\section{Three-dimensional vorticity distribution}

For the three-dimensional case, we must discuss an appropriate form for the spatial distribution of vorticity. Just as the vorticity in two dimensions was discretized to a collection of $N$ delta functions or smooth core structures, the vorticity in three dimensions is often discretized in the form of vector valued vortex particles or vortex filaments. ${ }^{1}$ Considering the vector particle approach, the vorticity is assumed to be highly concentrated only at discrete locations $\mathbf{x}_{i},{ }^{23}$

$$
\boldsymbol{\omega}(\mathbf{x}, t)=\sum_{i}^{N} \boldsymbol{\alpha}_{i} \gamma_{i}\left(\mathbf{x}-\mathbf{x}_{i}(t)\right)+\hat{\boldsymbol{\omega}}(\mathbf{x}, t),
$$

where the vector $\boldsymbol{\alpha}_{i}$ has the units of circulation times length, and the spatial distribution for each vortex is given by some function $\gamma_{i}(\mathbf{x})$,

$$
\gamma_{i}(\mathbf{x})=\frac{1}{\sigma_{i}^{3}} p\left(|\mathbf{x}| / \sigma_{i}\right)
$$

with an effective core radius $\sigma_{i}$. In the case $\sigma_{i}=0$, the $\gamma_{i}$ 's become Dirac delta functions. The term $\hat{\boldsymbol{\omega}}(\mathbf{x}, t)$ in Eq. (10) represents the additional term needed to ensure the divergence free requirement. ${ }^{23}$ It will not be considered further, since it makes no contribution to the velocity field. The above vorticity distribution can be substituted into the BiotSavart equation in three dimensions,

$$
\mathbf{u}(\mathbf{x})=-\frac{1}{4 \pi} \int \frac{\left(\mathbf{x}-\mathbf{x}^{\prime}\right) \times \boldsymbol{\omega}\left(\mathbf{x}^{\prime}\right)}{\left|\mathbf{x}-\mathbf{x}^{\prime}\right|^{3}} d \mathbf{x}^{\prime},
$$

to obtain a discrete form of the velocity induced by the $N$ vortex elements. Note that in this representation both the positions of vortex elements and their intensities $\boldsymbol{\alpha}_{i}$ can change. Assuming ergodicity in the phase space, velocity induced by one vortex element is characterized by some mean intensity $\overline{\boldsymbol{\alpha}}$ which is the same for all vortex elements.

Following a derivation similar to section II A, in the singular case we obtain $p_{i}\left(\left|\mathbf{u}_{i}\right|\right) \sim\left|\mathbf{u}_{i}\right|^{-5 / 2}$. For the desingularized (finite $\sigma_{i}$ ) case, we have the same tail, but there is a maximum cut-off velocity.

For the case where the three-dimensional vorticity is represented by thin vortex filaments the results of the twodimensional analyses can be applied. This is because as $\mathbf{x}$ nears the filament, the high velocity tail approaches the form of the two-dimensional vortex case. For the sake of convenience, we shall henceforth omit derivations involving filaments with the implicit understanding that the results of the two-dimensional analyses carries over directly. This also has the interesting implication that the velocity statistics can have different behavior depending on the physical form of the vorticity. 


\section{PDF of the velocity difference}

Now we apply our methods to investigate tails of the probability distribution for the velocity difference $p_{\delta \mathbf{u}}(\delta \mathbf{u}(\mathbf{x} ; \delta \mathbf{r}))$, where $\delta \mathbf{u}(\mathbf{x} ; \delta \mathbf{r})=\mathbf{u}(\mathbf{x}+\delta \mathbf{r})-\mathbf{u}(\mathbf{x})$. We decompose the velocity difference as

$$
\delta \mathbf{u}(\mathbf{x}, \delta \mathbf{r})=\sum_{i=1}^{N} \delta \mathbf{u}_{i}(\mathbf{x}, \delta \mathbf{r}),
$$

where the contribution of each vortex is

$$
\delta \mathbf{u}_{i}(\mathbf{x} ; \delta \mathbf{r})=-\frac{\Gamma_{i}}{2 \pi}\left[\frac{\left(\mathbf{x}-\mathbf{x}_{i}\right) \times \hat{\mathbf{e}}_{z}}{\left|\mathbf{x}-\mathbf{x}_{i}\right|^{2}}-\frac{\left(\mathbf{x}+\delta \mathbf{r}-\mathbf{x}_{i}\right) \times \hat{\mathbf{e}}_{z}}{\left|\mathbf{x}+\delta \mathbf{r}-\mathbf{x}_{i}\right|^{2}}\right] .
$$

Expanding for small $\delta r=|\delta \mathbf{r}|$, we have

$$
\left|\delta \mathbf{u}_{i}(\mathbf{x} ; \delta \mathbf{r})\right| \approx \frac{\Gamma_{i}}{2 \pi} \frac{\delta r}{\rho_{i}^{2}} .
$$

Using a derivation similar to section II A, for the same sign and magnitude $\Gamma_{i}$, we have

$$
P\left(\left|\delta \mathbf{u}_{i}\right|>\delta U\right) \sim \int_{B} p\left(\mathbf{x}_{1}\right) \delta \mathbf{x}_{1} \sim \rho^{2} \sim \delta U^{-1},
$$

and therefore

$$
p_{i}\left(\left|\delta \mathbf{u}_{i}\right|\right) \sim\left|\delta \mathbf{u}_{i}\right|^{-2}, \quad \text { for large }\left|\delta \mathbf{u}_{i}\right| .
$$

For the three-dimensional particle case,

$$
P\left(\left|\delta \mathbf{u}_{i}\right|>\delta U\right) \sim \int_{B} p\left(\mathbf{x}_{1}\right) \delta \mathbf{x}_{1} \sim \rho^{3} \sim \delta U^{-1},
$$

where $B$ is now a ball in three-dimensions of radius $\rho$, we see that we obtain the same result for the tails of the PDF, i.e. (17) holds. Similar derivations can be made for velocity derivatives.

In the case $\delta r \gg \rho$, in two-dimensions, it is easy to see that the tail of the PDF is the same as in the case of the velocity $\left(|\delta \mathbf{u}|^{-3}\right)$, as contributions to the tail arise from the time that the vortex spends in a small neighborhood of any of the two points involved in the difference. In threedimensions, by the same argument, the tail has a $|\delta \mathbf{u}|^{-5 / 2}$ decay for large $\delta r$.

\section{LIMIT DISTRIBUTIONS}

In this section we will consider sums of independent random variables for which tails of PDFs decay algebraically. In the previous section we considered the norm of the velocity $\mathbf{u}$ and velocity difference $\delta \mathbf{u}$. We chose those quantities because of the ease of the presentation (rotational symmetry). The same results on the tails hold for the velocity components. For the calculation of limits in this section we consider the PDF $p_{u}(u, \mathbf{x})$ of the velocity component $u$ and the PDF $p_{\delta u}(\delta u, \mathbf{x})$ of the velocity difference component $\delta u$ in the direction of arbitrary axis. We do this again for the clarity of presentation, as symmetry of the single vortex PDF $p_{i}\left(u_{i}, \mathbf{x}\right) \quad\left(p_{\delta u}\left(\delta u_{i}, \mathbf{x}\right)\right)$ when $u_{i} \rightarrow+\infty \quad\left(\delta u_{i} \rightarrow+\infty\right)$ and $u_{i} \rightarrow-\infty\left(\delta u_{i} \rightarrow-\infty\right)$ can be used. Similar results (with nonsymmetric stable distributions) can be obtained for the norm of the velocity, velocity difference and velocity derivatives.
Since we are interested in the PDFs of the sums of random variables $u_{i}$ or $\delta u_{i}$, we turn to limit distribution theorems to find the forms of the PDFs. We will first briefly discuss the central limit theorem, and then go on to other stable distributions.

In the study of normalized sums of independent random variables of the form

$$
X_{N}=\frac{1}{\sqrt{N}} \sum_{i=1}^{N} X_{i},
$$

where $X_{i}$ 's have a common PDF $p_{i}\left(x_{i}\right)$, the central limit theorem establishes the conditions under which $X_{N}$ is asymptotically normally distributed. In its simplest form, the central limit theorem states ${ }^{11}$ that for a system with mean $E\left(X_{i}\right)=0$ and variance $\sigma_{i}^{2}=1$, as $N \rightarrow \infty$ the distribution of the normalized sum $X_{N}$ tends to the normal distribution with the density

$$
p(x)=\frac{1}{\sqrt{2 \pi}} e^{-x^{2} / 2} .
$$

As applied to our $N$-vortex problem, if the velocity contribution of each vortex $u_{i}(\mathbf{x})$ can be considered an independent, identically distributed random variable, then as $N \rightarrow \infty$, $p_{u}(u ; \mathbf{x})$ approaches a normal distribution regardless of the shape of the individual density function $p_{u_{i}}\left(u_{i}\right)$, provided the mean and the variance exist, as we can always shift the mean to zero and scale the variance to 1 . This is the case for vortex blobs, where the finite velocity insures existence of the mean and the variance.

In general, Lévy stable distributions are interesting because of the fact that they are the only possible limiting probability distributions of normed sums of stationary independent random variables,

$$
X_{N}=\frac{1}{B_{N i}} \sum_{i}^{N} X_{i}-A_{N}
$$

(see the introductory section or Refs. 11, 24, 15 or 25 for an interesting example in stellar dynamics). Because of the above mentioned symmetry of distributions induced by single vortices we are only going to be interested in symmetric Lévy stable distributions that have characteristic functions of the form

$$
\phi_{i}(k)=e^{-a|k|^{\alpha}}, 0<\alpha \leqslant 2 .
$$

The conditions on the range of $\alpha$ are imposed by the fact that $\int p(u) d u=1$. The $\alpha$ 's are known as the characteristic exponents, with $\alpha=2$ being the Gaussian distribution and $\alpha=1$ being the Cauchy distribution. Apart from being well known, these two special cases are also of particular interest to our application (as is $\alpha=3 / 2$, the Holtzmark distribution). For large $x$ and $0<\alpha<2$, the PDF with a characteristic function (20) has the asymptotic $(|x| \rightarrow \infty)$ form (see Ref. 13)

$$
p(x) \sim \frac{\Gamma(\alpha) \sin (1 / 2) \pi \alpha}{\pi|x|^{\alpha+1}}, \quad 0<\alpha<2 .
$$

Now, consider the random variable $X$ with zero mean and distribution function $F(x)=P(X<x)$. Following Ref. 24, if 
TABLE I. Summary of PDFs.

\section{2-D}

3-D

$p_{u}(u)$

$p_{\delta u}(\delta u)$ small $\delta r$

$p_{\delta u}(\delta u)$ large $\delta r$

normal $(\alpha=2)$

normal $(\alpha=2)$
Cauchy $(\alpha=1)$
Holtzmark $(\alpha=3 / 2)$

Cauchy $(\alpha=1)$

Holtzmark $(\alpha=3 / 2)$
$F(x) \sim c / x^{\alpha}$ as $x \rightarrow-\infty$ and $F(x) \sim 1-c / x^{\alpha}$ as $x \rightarrow \infty$, where $0<\alpha<2, c>0$ then $F$ belongs to the domain of attraction of a symmetric stable Lévy distribution with exponent $\alpha$. In other words, the sum (19) where each $X_{i}$ is identically distributed as $X, A_{N}=0$ and $B_{N}=N^{1 / \alpha}$ converges in distribution to a Lévy stable distribution with exponent $\alpha$. In the case when $\alpha=2$ the limiting distribution is Gaussian. Based on this and the results in section II, in the case of a two-dimensional velocity field we expect convergence towards the Gaussian. It is interesting to point out that the variance for a system of vortices in 2-D diverges logarithmically! Still, the limiting distribution is Gaussian (as the logarithmic function is "slowly varying;' see Ref. 24). This is important for the very slow convergence to a Gaussian that we observe numerically (see section V). For threedimensional vortex particles, $\alpha=3 / 2$, and the convergence towards the Holtzmark distribution should be seen. These two results have already been discussed by Takayasu. ${ }^{14}$ As mentioned earlier, for the case of vortex filaments in three dimensions, the limiting distribution for the velocity is Gaussian, following the two dimensional result.

For the velocity difference distribution in the case of a small separation $\delta r$, in both two and three dimensions, the $\delta u^{-2}$ tail for the PDF gives $\alpha=1$, and a Cauchy distribution. In the case of the large separation $\delta r$, in two dimensions the tail $\delta u^{-3}$ gives a Gaussian distribution, while in three dimensions we obtain Holtzmark distribution. The velocity and velocity difference PDFs for the singular cases can be summarized as shown in Table I. It is important to note that a system of vortex blobs will tend toward the normal distribution because of the finite variance. The rate of approach toward the normal distribution will be different for velocity and velocity derivatives, as the tails coming from the influence of a single vortex are different. For example, for small $\delta_{c}$ the PDF for the velocity component induced by all vortices, $p_{u}(u)$ will approach the normal distribution more rapidly as $N$ increases, compared to the PDF for the velocity difference component $p_{\delta u}(\delta u)$. This retention of the powerlaw form for $p_{\delta u}(\delta u)$ is a consequence of the fact that, having the velocity cutoff $\delta_{c}$, the tails for the velocity PDF are going to extend to the value of velocity of the order $1 / \delta_{c}$, while the tails of the PDF of the velocity difference reach values of the velocity difference of the order $1 / \delta_{c}^{2}$. An interesting question is: what is the limiting distributions for the velocity difference for intermediate values of the vortex smoothing parameter $\delta_{c}$, and for intermediate values of the separation parameter $\delta r$. These parameters are related in the sense that varying one or the other varies the inter-vortex distance versus the separation distance between the velocity measuring points. Although there is no strict limiting theorem for intermediate values, the distribution has to make a smooth transition from Cauchy (concave in a semi-log plot) to Gaussian (convex in a semi-log plot), starting at the tails, as the vortex core is increased, or as the separation distance $\delta r$ is increased. We believe the intermediate distribution passes through the exponential and near exponential distributions. These issues are discussed further in the next section and in section $\mathrm{V}$.

The above derivation is valid for a system of $\mathrm{N}$ vortices, each of which has the same sign and magnitude of circulation $\Gamma_{i}$ under the assumptions outlined in section II. Note that the condition of having vortices with identical circulation may be difficult to meet in a real physical situation, since vortices of different magnitudes may be found in a given flow. However, we expect our conclusions to hold for a system of $N$ vortices where the average circulation $\bar{\Gamma}$ is equal to $\Gamma$ for a system considered here, if the differences between magnitudes of circulation of vortices are not too large. Departure from the above described behavior is also expected for small $N$.

\section{COMPARISON TO SOME EXPERIMENTAL RESULTS}

In the introductory section, we briefly discussed some of the experimental and numerical evidence regarding the velocity difference PDF in turbulent flows. Recent numerical experiments have shown that the small scales of turbulent flows are composed of a collection of discrete vortex elements. ${ }^{3}$ The velocity difference defined as $\delta u(\mathbf{x}, \delta \mathbf{r}) \equiv u(\mathbf{x}+\delta \mathbf{r})-u(\mathbf{x})$ has often been measured, along with the velocity $u(\mathbf{x})$, to characterize turbulent flow. These measurements typically show the velocity PDFs of nearnormal shape, and velocity difference PDFs of nearexponential shape, or, as in Tong and Goldburg's work, Cauchy shape. While the near-normality of the PDF of the velocity can be explained in terms of the central limit theorem, ${ }^{26,27}$ several explanations have been put forth for the near-exponential shape of the velocity difference PDF. 3,28,29 Frisch and $\mathrm{She}^{30}$ have proposed a similarity argument that allows a nonlinear transformation between the variables $u_{0}$ and $s=\partial u / \partial x$ so that $p_{s}(s)$ can be expressed as a function of $p_{u_{0}}\left(u_{0}\right)$. A normally distributed $p_{u_{0}}\left(u_{0}\right)$ produces a stretched exponential function (to the $4 / 3$ power) for $p_{s}(s)$. Extensions of this argument to include various intermittency (K62 and multifractal) models have also been made. ${ }^{30,31}$ Kraichnan's model produces a strict exponential for $p_{s}(s){ }^{32}$ Still another model is due to $\mathrm{Kida}^{33}$ in which he proposes a "log-stable" model that also produces a stable distribution for the $\log$ of the dissipation field.

Experimental studies of turbulent flows using photon correlation spectroscopy by Tong and Goldburg ${ }^{7-9}$ (see also Ref. 34) have shown that the distribution of relative velocity (difference) is well approximated by the product of a Lorenztian (Cauchy) function and a Gaussian-like function. They suggest that the two functions are due to the effects of two distinct regions of turbulent flow, with the small fluctuations being characterized by the Lorentzian function, and the large fluctuations being characterized by the Gaussian function. In the previous section, we have shown that the PDF of the 
velocity difference (for small $r$ ) converges towards the Cauchy distribution,

$$
p_{\delta u}(\delta u)=\frac{1}{\pi} \frac{c}{c^{2}+\delta u^{2}}
$$

for an ergodic system of discrete vortices in three dimensions.

As the vortex smoothing parameter $\delta_{c}$ increases, the distribution departs from Cauchy starting at the tails. That is, the effect of smoothing of the vorticity is to produce Gaussian tails at larger $|\delta u|$ in the PDF. Thus, Tong and Goldburg's contention that $p_{\delta u}(\delta u)$ has a Cauchy distribution for small relative velocity fluctuations, and near-Gaussian for large relative velocity fluctutations could follow from an argument similar to the ones presented in the previous sections. The Cauchy distribution derives from the power-law fall off $\left[\delta u(\rho) \sim 1 / \rho^{2}\right.$ for $2-\mathrm{D}, \sim 1 / \rho^{3}$ for $\left.3-\mathrm{D}\right]$ of the velocity difference outside the vortex blob core region. This contributes to the central (small $|\delta u|$ ) region of $p_{\delta u}(\delta u)$. The tails of $p_{\delta u}(\delta u)$ are determined by the large $|\delta u|$ behavior, which is a function of the shape of the vortex core. However, because of the cutoff, it is reasonable to assume that a Gaussian tail will result. Note that this explanation is in complete accordance with Onuki's ${ }^{34}$ discussion of Tong and Goldburg's experiments, and gives his arguments a simplified physical model (a system of $N$ vortex elements) as a base. $\left(\right.$ Onuki $^{34}$ has previously suggested an approach similar to ours in reference to stable distributions and Takayasu's ${ }^{14}$ study.)

We note that although the most commonly observed velocity difference PDF in turbulence is the one with exponential tails, there is actually quite a bit of variability in the measurements of the PDFs with respect to the separation parameter $\delta r$, as illustrated by Figure 1 in Anselmet et al.'s paper $^{28}$ or in Figures 7 and 8 in Vincent and Meneguzzi's paper. ${ }^{3}$ The variation of $\delta r$ corresponds to probing of different spatial scales of turbulence (inertial range and dissipation range, for example). Onuki ${ }^{34}$ attributes the contrasting results of Tong and Goldburg to the greater Reynolds number in their experiments, but there is a difference in experimental procedure employed by Tong and Goldburg and that of other authors. In particular, Tong and Goldburg's measurements which use the homodyne method, give the intensity of scattered light correlation function which depends on the velocity difference of all particle pairs in the scattering volume. For these pairs, $\delta r$ ranges from dissipative scale to integral scale. Thus, the measurements give an average of velocity difference over dissipative, inertial and integral scales. We have shown analytically in our model that for small $\delta r$, for small velocity fluctuations and for small $\delta_{c}$ in three dimensions the PDF of velocity differences is Cauchy. Also, we have shown that under the same conditions, for large $\delta r$ the PDF is Gaussian. It is numerically shown in the next section (and qualitatively argued in the previous section) that exponential tails can arise for intermediate values of $\delta r$. It is easy to imagine that averaging over different $\delta r$ can produce a PDF that looks like a product of a Cauchy and a Gaussian with a transitional exponential region. In experiments by other authors, $\delta r$ is typically chosen in the inertial range and fixed. Exponential tails are typically observed, but, as mentioned above, there is variability with $\delta r$.

Another aspect of turbulent flow statistics that this model is consistent with is the fact that the flatness of the distribution of spatial derivatives increases as the order of the velocity derivative increases. ${ }^{26,36}$ In three dimensions,

$$
\begin{aligned}
& \frac{\partial^{n} u}{\partial \rho^{n}} \sim \rho^{-2-n}, \\
& P\left(\frac{\partial^{n} u}{\partial \rho^{n}}>U^{n}\right) \sim \rho^{3}, \\
& p\left(\frac{\partial^{n} u}{\partial \rho^{n}}\right) \sim\left(\frac{\partial^{n} u}{\partial \rho^{n}}\right)^{-3 /(n+2)-1} .
\end{aligned}
$$

Therefore, as $n$ increases, the distribution becomes flatter, implying strong intermittency.

\section{NUMERICAL SIMULATIONS}

We now provide numerical results for 2-D systems of vortex elements, and compare them with the analytical discussion in previous sections. We will see that the numerical simulations seem to justify the assumptions that we made in order to derive limiting distributions.

The velocity field due to $N$ vortices in a stationary configuration was numerically studied using vortex methods. $^{37,22,1}$ The vortex method of flow computation involves keeping track of the vorticity $\omega$ as it moves around in a Lagrangian sense. The calculation of velocity is done by the Biot-Savart law, which for two-dimensions is

$\mathbf{u}(\mathbf{x}, t)=\frac{d \mathbf{x}}{d t}(\mathbf{x}, t)=-\frac{1}{2 \pi} \int \frac{\left(\mathbf{x}-\mathbf{x}^{\prime}\right) \times \boldsymbol{\omega}\left(\mathbf{x}^{\prime}, t\right)}{\left|\mathbf{x}-\mathbf{x}^{\prime}\right|^{2}} d \mathbf{x}^{\prime}+\nabla \phi$.

This is the solution to the Poisson equation describing the relationship between the velocity $\mathbf{u}$ and the vorticity $\boldsymbol{\omega}$,

$$
\nabla^{2} \mathbf{u}=-\nabla \times \boldsymbol{\omega} .
$$

The domain of motion in our problem is infinite with no solid boundaries and no flow at infinity which dictates that $\nabla \phi=0$. The nature of the same-sign-same-magnitudevortices flow is such that the motion actually takes place in a finite domain. In two dimensions the vorticity is $\boldsymbol{\omega}=\omega \hat{\mathbf{e}}_{z}$ and for the point vortex case the scalar field $\omega$ is represented by Eq. (1),

$$
\omega(\mathbf{x}, t)=\sum_{i=1}^{N} \Gamma_{i} \delta\left[\mathbf{x}-\mathbf{x}_{i}(t)\right]
$$

where $\delta$ is the Dirac delta function and $\Gamma_{i}$ is the strength of the $i$ th vortex. This gives a system of $2 N$ nonlinear ODEs for the positions of the $N$ vortices:

$$
\frac{d \mathbf{x}_{i}}{d t}=-\frac{1}{2 \pi} \sum_{j=1, j \neq i}^{N} \frac{\Gamma_{j}\left(\mathbf{x}_{i}-\mathbf{x}_{j}\right) \times \hat{\mathbf{e}}_{z}}{\left|\mathbf{x}_{i}-\mathbf{x}_{j}\right|^{2}} .
$$

Because of the singularity at the core of the vortices, vortex blob methods are often used to simulate vortical flows. There are several ways to de-singularize the 
vorticity, ${ }^{22,23}$ but we use the simple algebraic core method with the parameter $\delta_{c}$. The vorticity distribution is now given by Eq. (8),

$$
\omega(\mathbf{x}, t)=\frac{1}{\pi} \sum_{i=1}^{N} \frac{\Gamma_{i} \delta_{c}^{2}}{\left(\delta_{c}^{2}+\left|\mathbf{x}-\mathbf{x}_{i}\right|^{2}\right)^{2}}
$$

and the velocity of each vortex blob evaluated at the center is

$$
\frac{d \mathbf{x}_{i}}{d t}=-\frac{1}{2 \pi} \sum_{j=1, j \neq i}^{N} \frac{\Gamma_{j}\left(\mathbf{x}_{i}-\mathbf{x}_{j}\right) \times \hat{\mathbf{e}}_{z}}{\left|\mathbf{x}_{i}-\mathbf{x}_{j}\right|^{2}+\delta_{c}^{2}} .
$$

The parameter $\delta_{c}$ can then be conveniently used to vary the vorticity distribution from isolated point vortices $\left(\delta_{c}=0\right)$ to vortices having significant overlap ( $\delta_{c}=$ large). Along with the parameter $N$, this allows us to explore the consequences of different vorticity distributions in terms of the velocity statistics.

There are four known invariants for the singular vortex system: ${ }^{22}$

$x$-linear impulse, $\quad I_{x}=\sum_{i=1}^{N} \Gamma_{i} x_{i}=$ const;

$y$-linear impulse, $\quad I_{y}=\sum_{i=1}^{N} \Gamma_{i} y_{i}=$ const;

angular impulse, $\quad I_{r^{2}}=L^{2}=\sum_{i=1}^{N} \Gamma_{i} r_{i}^{2}=$ const;

and

$$
\text { energy, } \quad \begin{aligned}
I_{E}=H & =-\frac{1}{4 \pi} \sum_{i \neq j}^{N} \sum_{j \neq i}^{N} \Gamma_{i} \Gamma_{j} \log \left|\mathbf{r}_{i}-\mathbf{r}_{j}\right| \\
& =\text { const },
\end{aligned}
$$

and similarly for the vortex blobs. Solutions of the vortex motion must observe the invariance of these quantities.

Equation (28) or (29) is integrated using a fourth order Runge-Kutta scheme for the Lagrangian motion of the vortices. Velocity statistics are collected at stationary points in the domain. The time steps $(\Delta t)$ are chosen such that the change in the invariant $H$ [Eq. (33)] is maintained to within $1 \%$ of the initial value. The other invariants [Eqs. (30)-(32)] are satisfied to much higher levels of accuracy. While there have been numerical techniques developed (symplectic integrators, see Ref. 38 , for example) specifically to take advantage of the Hamiltonian structure of systems such as ours, they were deemed computationally economic only for cases where extremely stringent requirements on the invariance of $H$ were necessary. Because of the chaotic nature of the vortex motion, error propagation during numerical computation is inevitable. This is a clear manifestation of the "sensitive dependence on initial conditions," and there is no way around it. It is of course, helpful to maintain the known invariants of motion for vortex systems [see Eqs. (30)-(33)]. In the following we take the position that while the exact position of the vortices cannot be known, the overall properties remain the same and correct in the statistical sense.

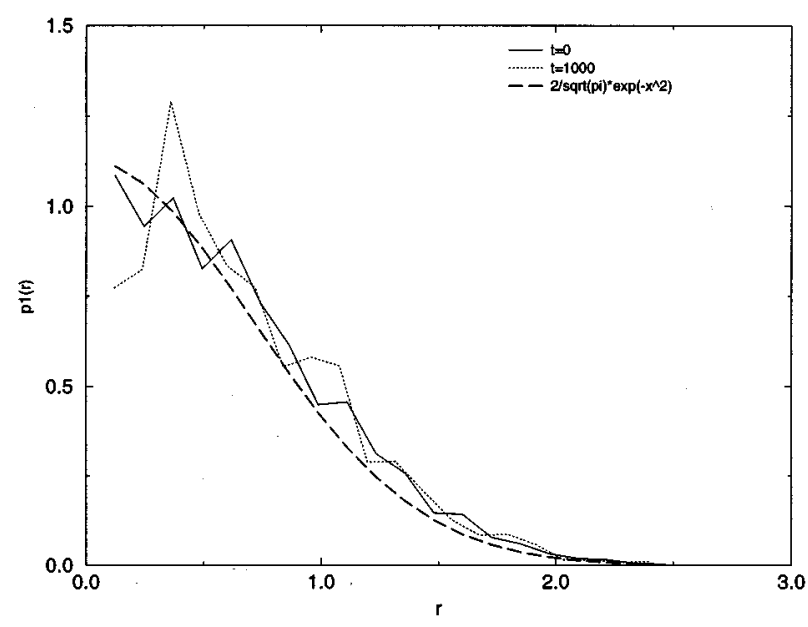

FIG. 1. $p_{1}(r)$ vs $r$ for $N=913, \delta=0.0033$ at $t=0$ and $t=1000$.

\section{A. Vortex configurations}

The simulations involve $N$ vortices that have the same circulation and sign (the purpose of this is to obtain long term statistics, as vortices of different signs tend to pair up and move away). The initial configurations are chosen such that they match the equilibrium state for the vortices as derived by Lundgren and Pointin. ${ }^{16}$

Although we roughly sketch Lundgren and Pointin's ${ }^{16}$ equilibrium statistics analysis of two-dimensional vortices below, it is recommended that the original article ${ }^{16}$ and other related papers by Montgomery and Joyce ${ }^{39}$ or Kraichnan and Montgomery ${ }^{40}$ for example be consulted. The reduced probability density function $p_{1}\left(\mathbf{x}_{1}\right)$ can be defined through the expression

$$
p_{1}\left(\mathbf{x}_{1}\right) d \mathbf{x}_{1}=\operatorname{Prob} .\left\{\mathbf{x}_{1} \in d \mathbf{x}_{1}\right\}
$$

and

$$
\int p_{1}\left(\mathbf{x}_{1}\right) d \mathbf{x}_{1}=1
$$

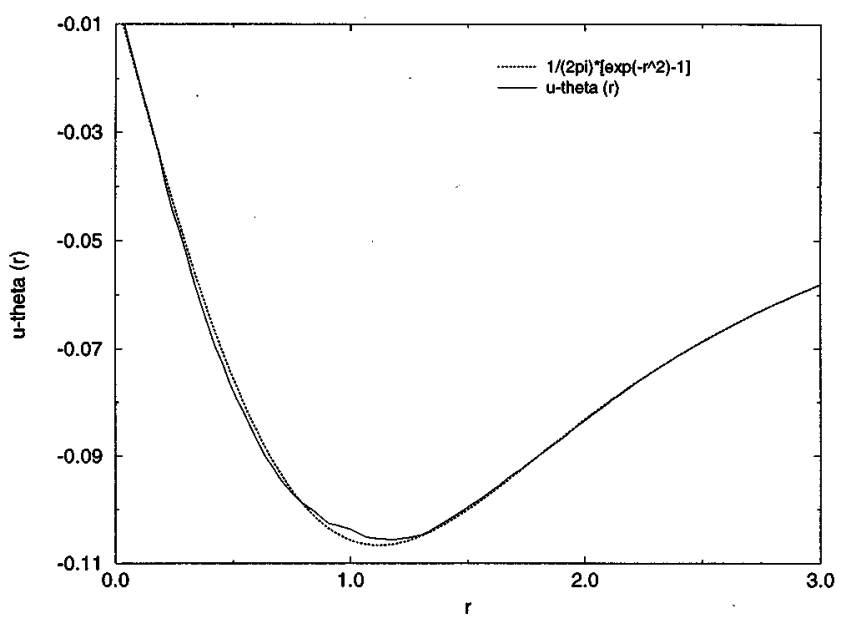

FIG. 2. $\left\langle u_{\theta}(r)\right\rangle$ for $N=913, \delta=0.0033$. 


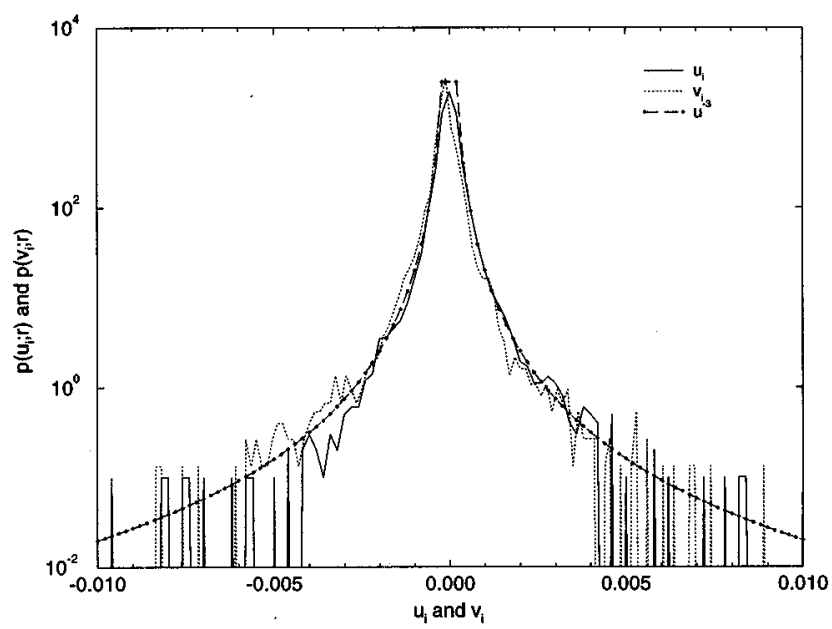

FIG. 3. $p_{u_{i}}\left(u_{i}\right)$ and $p_{v_{i}}\left(v_{i}\right)$ the PDF of the velocity due to a single vortex for $N=913, \delta_{c}=0$.

where $\mathbf{x}_{1}$ is the position of a single vortex. It follows naturally then, that the average vorticity, is given by

$$
\langle\omega(\mathbf{x})\rangle=\Gamma p_{1}(\mathbf{x}) .
$$

An equilibrium configuration based on a closure approximation for the one point distribution is then proposed, which produces an integral equation for $p_{1}\left(\mathbf{x}_{1}\right)$ that has the following Gaussian distribution as a solution for the parameter choice $\lambda=0$ (where $\lambda$ is proportional to the inverse temperature, related to the energy $H$ of the system),

$$
p_{1}\left(\mathbf{x}_{1}\right)=\frac{1}{\pi L^{2}} e^{-\left(\left(\mathbf{x}_{1}-I_{x} \hat{\mathbf{e}}_{x}-I_{y} \hat{\mathbf{e}}_{y}\right) / L\right)^{2}} .
$$

Since we will be dealing with vortex distributions that are symmetric about the origin (in the mean), we will use polar coordinates $(r, \theta)$, and represent the one point distribution $p_{1}\left(\mathbf{x}_{1}\right)$ by $p_{1}(r)$.

The initial vortex positions chosen for our numerical simulations are such that the invariants of motion are specified as follows [see Eqs. (30-33)]:

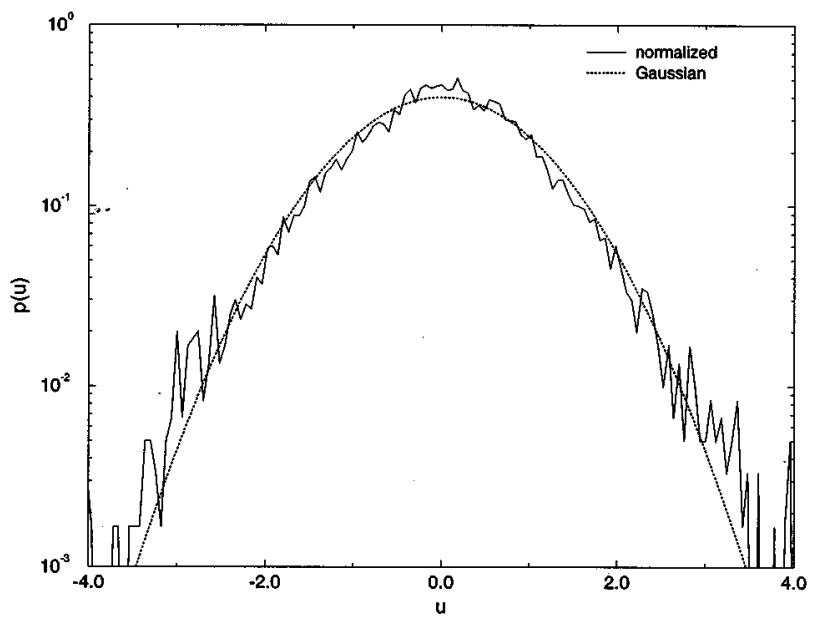

FIG. 4. Normalized $p_{u}(u)$ for $N=270, \delta_{c}=0.1$.

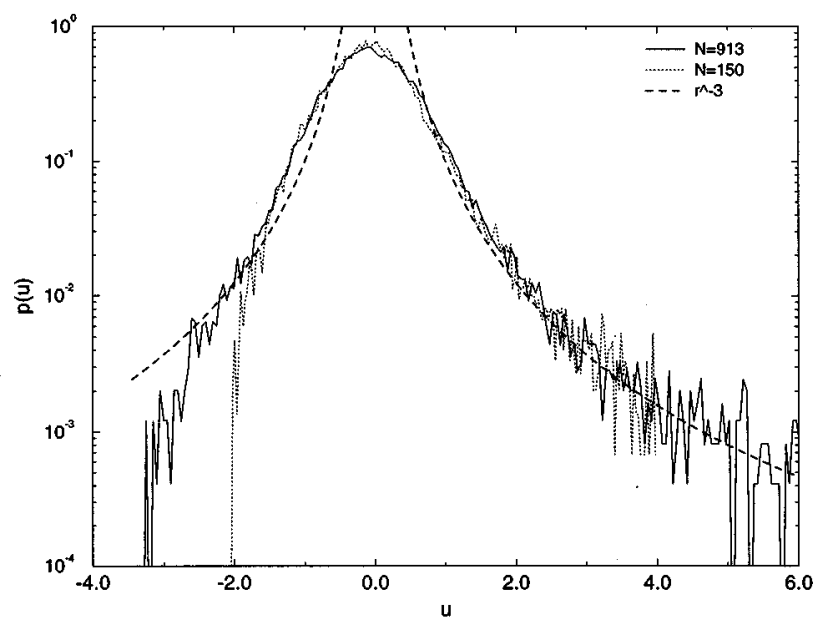

FIG. 5. Normalized $p_{u}(u)$ for $N=913$ and $N=150, \delta_{c}=0$.

$I_{x}=0, I_{y}=0, I_{r^{2}}=L^{2}=1, I_{E}=H=-0.00461$. This choice of the value for $H$ (energy) corresponds to Lundgren and Pointin's ${ }^{16} \lambda=0$ state with $p_{1}(r)$ given by $e^{-r^{2}} / \pi$. Since there are an infinite number of possible configurations for a fixed $H$, we randomly generate large numbers of initial configurations and pick one that is sufficiently close to the desired $H$. The $H$ values for the system can be "tweaked" by moving adjacent vortices closer to or farther away from each other (since the interaction energy is a function of the intervortex distances). All the vortices are of the same sign and magnitude, the total circulation $\Gamma$ satisfying $\Gamma=\sum_{i=1}^{N} \Gamma_{i}=1$.

The two conditions, $I_{r^{2}}=L^{2}=1$ and $\Gamma=\sum_{i=1}^{N} \Gamma_{i}=1$ together set the length scale and the time scale for the $N$-vortex system. All the other parameters $\delta_{c}, \delta r, x, y, t, u, v, \omega, H, \lambda$ are scaled with respect to the parameters $I_{r^{2}}=1$ and $\Gamma=1$.

Figure 1 shows typical probability densities $p_{1}(r)=\langle\omega(r)\rangle / \Gamma$ (see Ref. 16) at the beginning and end of the simulations with the predicted equilibrium curve. Our numerical simulations provide a limited validation of the clo-

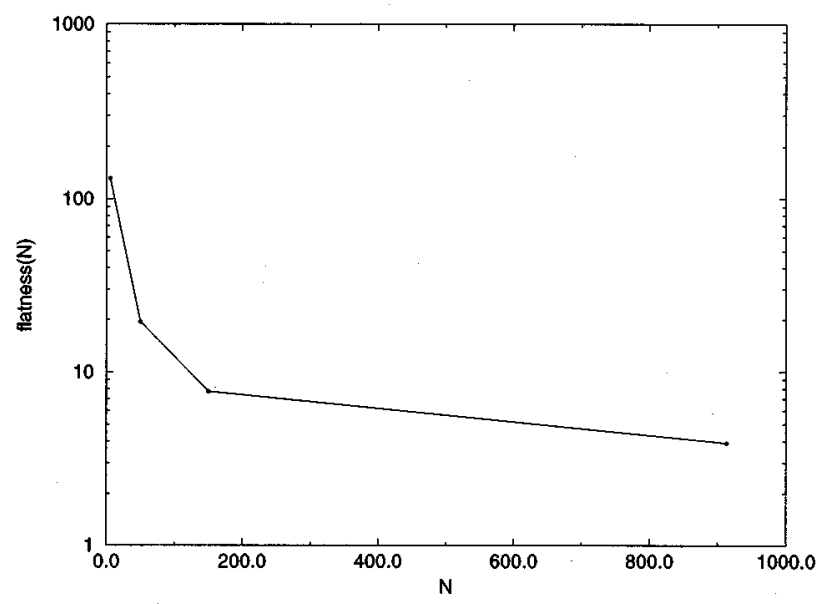

FIG. 6. Flatness $\mu_{4}$ as a function of $N$ for $\delta_{c}=0$, showing convergence towards the value 3 for large $N$. 


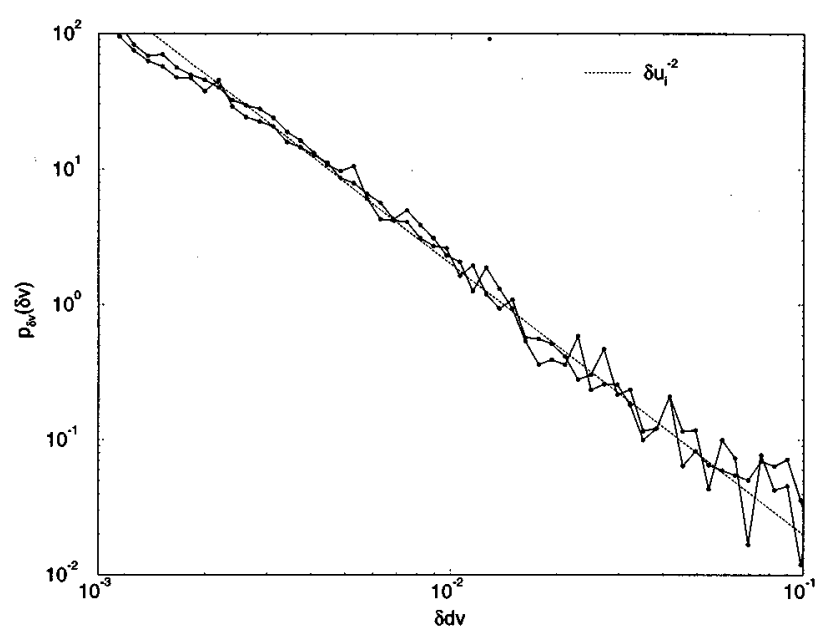

FIG. 7. $p_{\delta v_{i}}\left(\delta v_{i}\right)$, the PDF of velocity difference due to a single vortex, for $N=150, \delta_{c}=0.01, \quad \delta r=10^{-8}$.

sure scheme proposed by Lundgren and Pointin ${ }^{16}$ for the equilibrium vortex state, at least for initial configurations that start off with the predicted solution.

Assuming there is no $\theta$ dependence in the mean, the mean velocity can be evaluated by using Stokes' theorem,

$$
\int_{C} \mathbf{u} \cdot d \mathbf{l}=\int_{A} \omega d A
$$

and averaging over an ensemble of possible configurations,

$$
\left\langle u_{\theta}(r) 2 \pi r\right\rangle=\left\langle\int_{0}^{r} \omega 2 \pi r^{\prime} d r^{\prime}\right\rangle .
$$

Making use of Ref. 16,

$$
\begin{aligned}
& p_{1}(r)=\frac{1}{\pi} e^{-r^{2}}, \\
& \left\langle u_{\theta}(r)\right\rangle=\frac{\Gamma}{\pi r} \int_{0}^{r} e^{-r^{\prime 2}} r^{\prime} d r^{\prime}=-\frac{1}{2 \pi r}\left[e^{-r^{2}}-1\right] .
\end{aligned}
$$

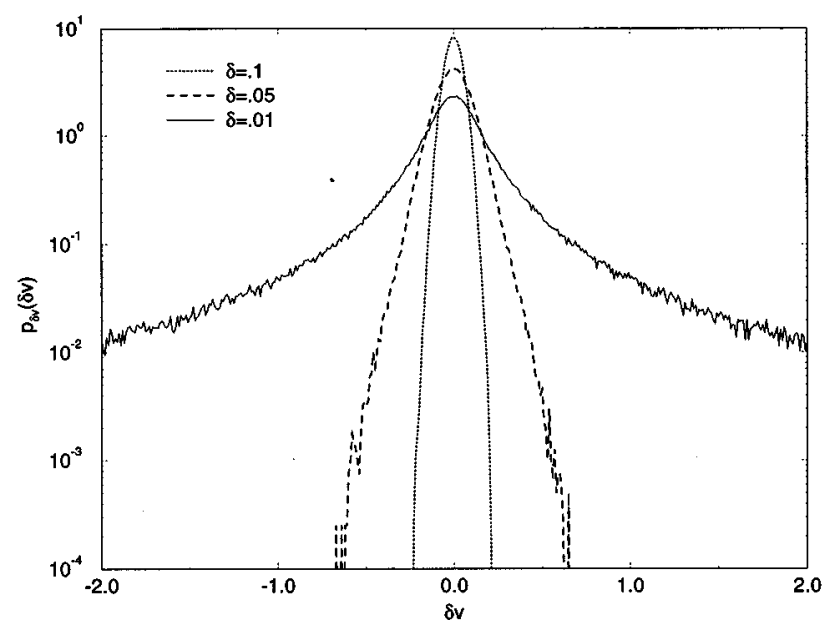

FIG. 8. $p_{\delta v}(\delta v)$, the PDF of the (normalized) velocity difference $\delta v=v_{1}(x+r)-v_{1}(x) / \delta r$ for various $\delta_{c}, N=150$.

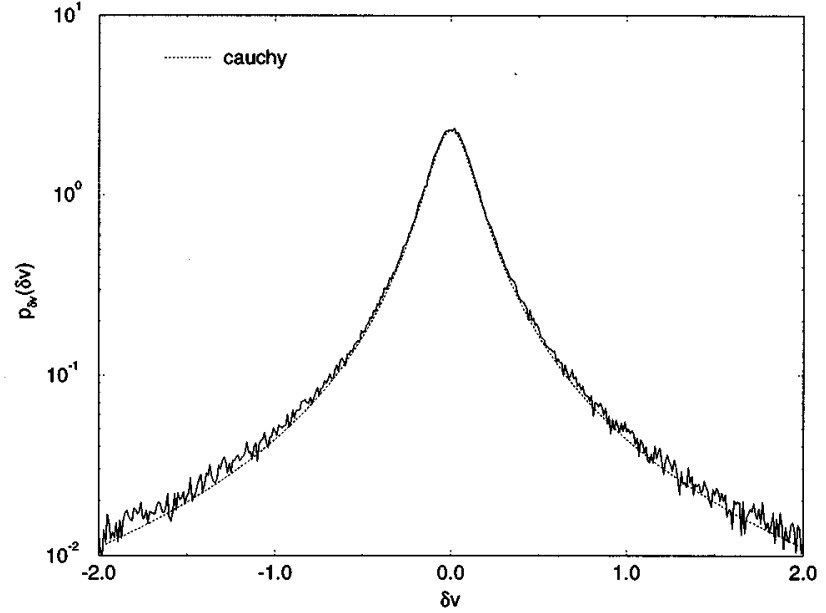

FIG. 9. $p_{\delta v}(\delta v)$, the PDF of the (normalized) velocity difference for $N=150, \delta_{c}=0.01, \delta r=10^{-8}$.

This average tangential velocity profile $\left\langle u_{\theta}(r)\right\rangle$ is compared with the results of a typical numerical simulation in Figure 2. For the numerical simulation, $\left\langle u_{\theta}(r)\right\rangle$ is computed at various $r$ along a fixed $\theta$, averaged over time (smoother statistics could of course have been obtained by averaging in $\theta$ direction as well). We are dealing with a non-homogenous flow, but when properly normalized, the PDFs are similar at any $\mathbf{x}$ location. For large $N$ and finite $\delta_{c}$, we can summarize the velocity field as consisting of a distinct large scale mean field, with statistical fluctuations about it. By statistical, we mean roughly that the law of large numbers is followed, which states that if the mean exists and the variance $\sigma_{i}^{2}$ (due to each vortex) is finite, then $\sigma^{2} \sim \sigma_{i}^{2} / N$. This implies that as $N \rightarrow \infty$, the fluctuations die out. This is seen in our simulations with vortex blobs.

\section{B. Numerical results}

The PDF $p_{u_{i}}\left(u_{i}\right)$ is plotted in Figure 3, along with a $u_{i}^{-3}$ power-law function for comparison. The normalized

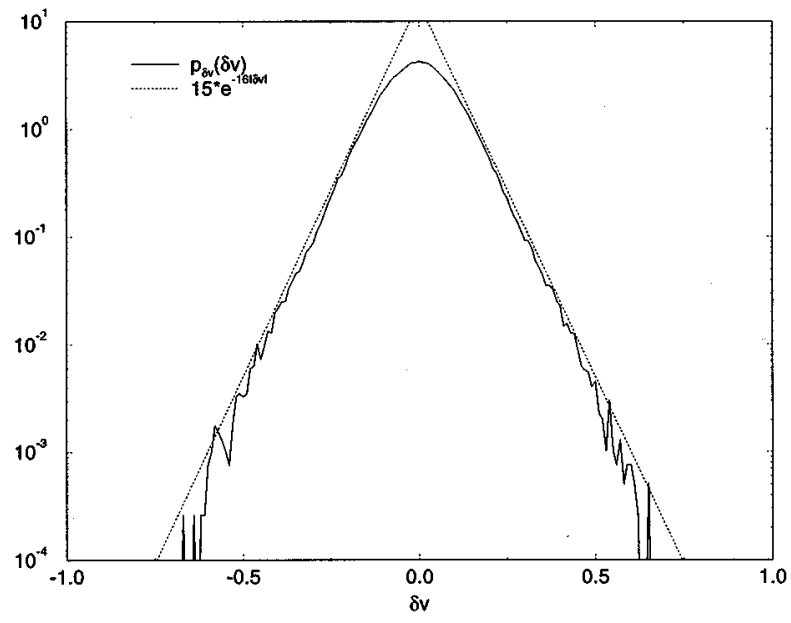

FIG. 10. $p_{\delta v}(\delta v)$, the PDF of the (normalized) velocity difference for $N=150, \delta_{c}=0.05, \delta r=10^{-8}$. 


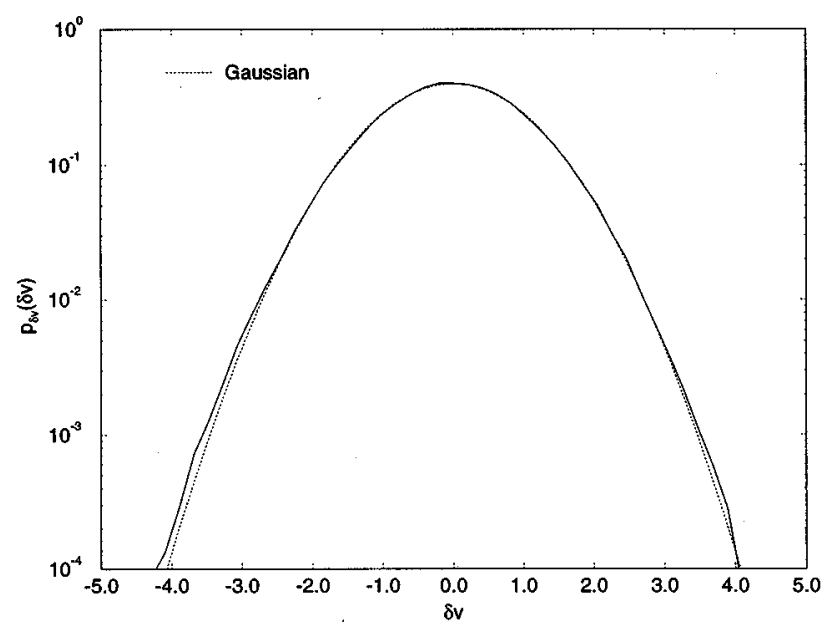

FIG. 11. $p_{\delta v}(\delta v)$, the PDF of the (normalized) velocity difference for $N=150, \delta_{c}=0.1, \delta r=10^{-8}$.

PDF $p_{u}(u)$ of $u=\Sigma u_{i}$, computed from a simulation with $\delta_{c} \neq 0$ is shown in Figure 4, along with a Gaussian curve. The fit is quite good, in contrast to the $\delta_{c}=0$ case shown in Figure 5. Our earlier derivation showed that $p_{u}(u)$ should converge towards the Gaussian distribution for the two dimensional case, however, $N$ needs to be very large before the convergence towards the Gaussian is noticeable. Figure 6 shows the flatness factor for various simulations with $\delta_{c}=0$ as $N$ is varied. Convergence towards a value of 3 (the Gaussian value) is seen as $N$ is increased. We have already mentioned (see section III) that this slow convergence can be explained in terms of the logarithmic divergence of the variance of a singular $N$-vortex system.

For the velocity difference, Figure 7 shows $p_{u_{i}}\left(\delta u_{i}\right)$, i.e., the contribution due to one vortex as it moves around the fixed measuring points. As predicted, there is a $\delta u_{i}^{-2}$ fall-off region in the middle, while the tails deviate because of the non-singular vortex core. Figure 8 shows $p_{\delta u}(\delta u)$ for several $\delta_{c}$ values (parameter values are as shown in the caption). We

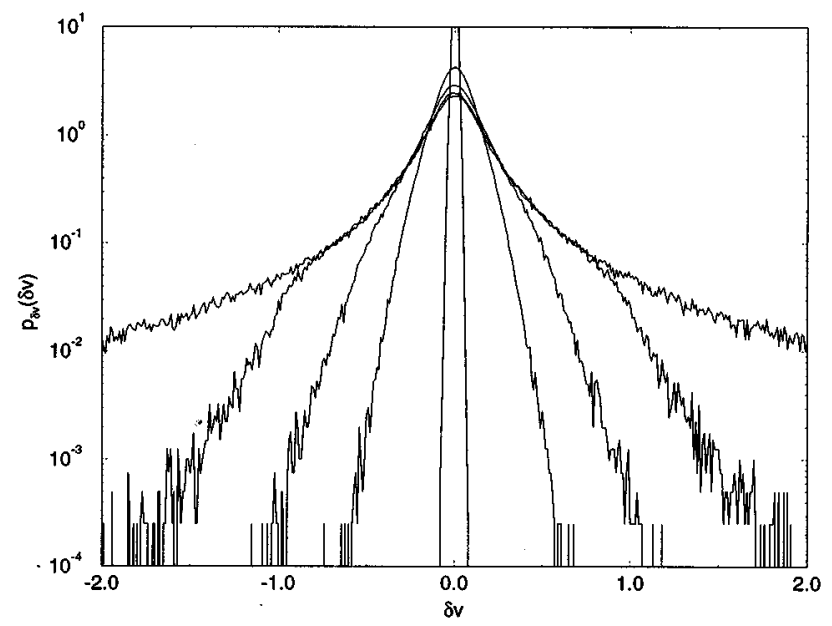

FIG. 12. $p_{\delta v}(\delta v)$, the PDF of the (normalized) velocity difference for $N=150, \delta_{c}=0.01$, and various $\delta r . \delta r=0.01,0.05,0.1,0.2,0.5$.

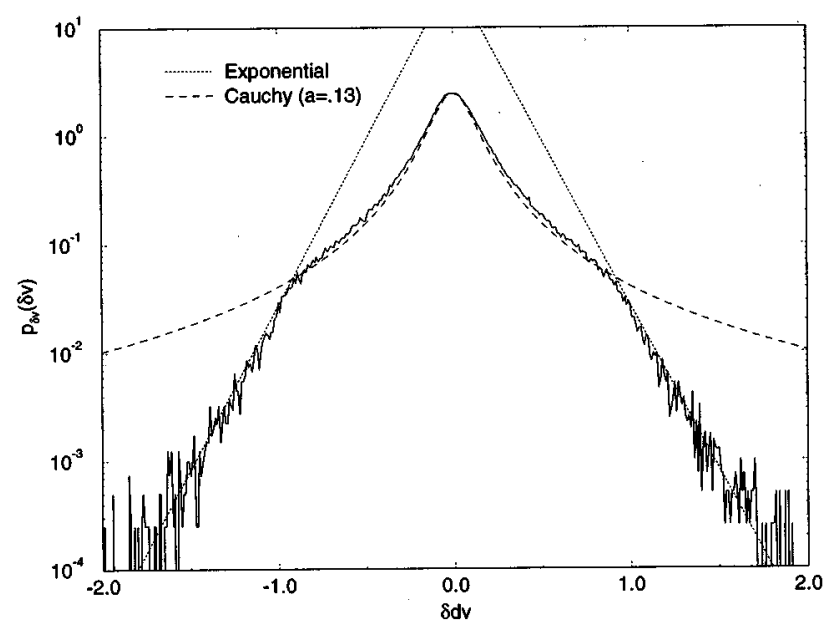

FIG. 13. $p_{\delta v}(\delta v)$, the PDF of the (normalized) velocity difference for $N=150, \delta_{c}=0.01, \delta r=0.05$

provide separate plots in Figures 9, 10, and 11, where the fit to Cauchy, exponential, and normal functions, respectively, are shown. A similar trend is seen for the variation of the separation parameter $\delta r$. For a fixed $\delta_{c}$ value, the Cauchy, exponential and normal distributions for $p_{\delta u}(\delta u)$ can be seen as $\delta r$ is increased. This can be seen in Figure 12. In particular, Figure 13 shows an intermediate $\delta r$ value at which the deviation from the Cauchy distribution can be seen occurring at the tails, and approaching the exponential distribution. Thus our numerical calculations are in agreement with the theory discussed in the earlier sections.

\section{SUMMARY}

We have described the velocity field statistics for the $N$ vortex problem, using a decomposition of the Eulerian velocity at a fixed point into a sum of $N$ individual components whose contributions are supplied by the velocity induced by each vortex. This allows us to make use of limit distribution theorems to describe the velocity at the fixed point. In particular, the tails of the velocity distribution of individual vortices have been used to show that there is a qualitative difference in the velocity statistics for the singular and the non-singular vortices. Vortex methods are also often used in modeling turbulent flows such as the mixing layer. The statistics collected from such simulations have to be carefully considered in light of the above discussion on the existence of the variance and the dependence of the variance on the value of $\delta_{c}$. The application of a similar methodology to the velocity difference produced distributions that shed some light on the possible source of the statistics that are seen in turbulent flow experiments. Numerical experiments involving two-dimensional vortex elements are presented; with results that match the theory well.

Note: It has come to our attention after the submission of this article, that results similar to those presented here have been independently arrived at by J. Jiménez [Abstracts of the APS DFD meeting, Irvine, California, November, 1995] in his study of two-dimensional turbulence. 


\section{APPENDIX: ERGODICITY AND VORTEX DENSITY DISTRIBUTIONS}

In this appendix we prove that ergodicity of the motion of the system of $N$ vortices of the same sign and circulation $\Gamma$ in the phase space $\mathbb{R}^{2 N}$ implies that $p_{i}\left(\mathbf{x}_{i}\right)=p_{j}\left(\mathbf{x}_{j}\right)$, for any $i, j$ in $\{0, \ldots, N\}$, where $p_{i}\left(\mathbf{x}_{i}\right)$ is the probability density function of $i$ th vortex [i.e., $\int_{A} p_{i}\left(\mathbf{x}_{i}\right) d A$ is a probability that the vortex $i$ is in the set $A$ in $\mathbb{R}^{2}$ or $\mathbb{R}^{3}$ ]. In other words, the one-vortex probability density is the same for all vortices, if the motion of the vortex system is ergodic.

Note first that $p_{i}\left(\mathbf{x}_{i}\right)$ is derived as a density of a set function $p($ vortex $i$ is in a set $A) \equiv p_{i}(A)$. We shall sometimes explicitly denote the dependence of this function on initial conditions of the vortex system by writing $p_{i}\left(A \mid\left(\mathbf{x}^{0}, \mathbf{y}^{0}\right)\right)$, where $\mathbf{x}_{0}=\left(x_{1}^{0}, \ldots, x_{N}^{0}\right), \mathbf{y}_{0}=\left(y_{1}^{0}, \ldots, y_{N}^{0}\right)$. If we show $p_{i}(A)=p_{j}(A)$ for all $i, j, A$, we are done.

Let $\phi^{t}\left(\mathbf{x}^{0}, \mathbf{y}^{0}\right)$ be the flow induced in $R^{2 N}$ by the system of $N$ vortices, depending on the initial conditions of vortices. By $\bar{A}$ we denote the "cylinder set" $A^{-}=\mathbb{R}^{2} \times \mathbb{R}^{2} \times \ldots \times A \ldots \times \mathbb{R}^{2}$ where $A$ is in the $i$-th position in the product. By $A^{\prime}$ we denote a cylinder set analogous to $\bar{A}$ with $A$ being in the $j$-th position in the product. Now, by definition, the probability that the vortex $i$ is in a set $A$ is the relative (compared to total time) amount of time that the vortex spends in $A$. Formally,

$$
\begin{aligned}
& p_{i}\left(A \mid\left(\mathbf{x}^{0}, \mathbf{y}^{0}\right)\right)=\lim _{t \rightarrow \infty} \frac{1}{t} \int_{0}^{t} I_{A}\left(\phi^{\bar{t}}\left(\mathbf{x}^{0}, \mathbf{y}^{0}\right)\right) d \bar{t}, \\
& p_{j}\left(A \mid\left(\mathbf{x}^{0}, \mathbf{y}^{0}\right)\right)=\lim _{t \rightarrow \infty} \frac{1}{t} \int_{0}^{t} I_{A^{\prime}}\left(\phi^{\bar{t}}\left(\mathbf{x}^{0}, \mathbf{y}^{0}\right)\right) d \bar{t},
\end{aligned}
$$

where $I_{X}$ is the indicator function for the set $X$, defined by $I_{X}(\mathbf{x}, \mathbf{y})=1$ if $(\mathbf{x}, \mathbf{y}) \in X$ and $I_{X}(\mathbf{x}, \mathbf{y})=0$ otherwise.

We define a symmetry $S$ by $S: x_{n} \rightarrow x_{n}, y_{n} \rightarrow y_{n}$ if $n$ $\neq i, j$ and $S: x_{i} \rightarrow x_{j}, x_{j} \rightarrow x_{i}, y_{i} \rightarrow y_{j}, y_{j} \rightarrow y_{i}$. Applying the symmetry $S$ is equivalent to interchanging positions of two vortices. The equations of motion for an $N$-vortex system are invariant under $S$, as the vortices are of the same sign and circulation. Thus,

$$
p_{i}\left(A \mid\left(\mathbf{x}^{0}, \mathbf{y}^{0}\right)\right)=p_{j}\left(A \mid S\left(\mathbf{x}^{0}, \mathbf{y}^{0}\right)\right) .
$$

This is easy to understand, as if we interchange the positions of two identical vortices $i$ and $j$ initially $(t=0)$, the motion is going to proceed in exactly the same manner, with the exception that the vortex $i$ is going to trace the trajectory of the vortex $j$ and vice versa.

Assuming the vortex system is ergodic implies that for almost all initial $\left(\mathbf{x}^{0}, \mathbf{y}^{0}\right)$ on the surface in the phase space defined by the constants of motion of the vortex system, $p_{i}\left(A \mid\left(\mathbf{x}^{0}, \mathbf{y}^{0}\right)\right)=c_{1}$, some constant. Also, for almost all initial conditions, $p_{j}\left(A \mid\left(\mathbf{x}^{0}, \mathbf{y}^{0}\right)\right)=c_{2}$. Assuming $c_{1} \neq c_{2}$ in the light of (A2) gives us a contradiction.

This proposition is not true in the case when vortices have the same sign but different circulations. For that case, and some consequences, see Ref. 41.

\footnotetext{
${ }^{1}$ A. Leonard, "Computing three-dimensional incompressible flows with
}

vortex elements,"' Annu. Rev. Fluid Mech. 17, 523 (1985).

${ }^{2}$ P. D. Koumoutsakos, "Direct numerical simulations of unsteady separated flows using vortex methods," Ph.D. thesis, California Institute of Technology, 1993.

${ }^{3}$ A. Vincent and M. Meneguzzi, "The spatial structure and statistical properties of homogeneous turbulence,"' J. Fluid Mech. 225, 1 (1991).

${ }^{4}$ W. J. A. Dahm, K. B. Southerland, and K. A. Buch, "Direct, highresolution, four-dimensional measurements of the fine scale structure of Sc $\gg 1$ molecular mixing in turbulent flows," Phys. Fluids A 3, 1115 (1991).

${ }^{5} \mathrm{P}$. G. Saffman, "Vortex interaction and coherent structures in turbulence," Transition and turbulence, Proceedings of a Symposium Conducted by the Mathematics Research Center, the University of Wisconsin-Madison, 13-15 October, edited by Richard E. Meyer, 1981.

${ }^{6}$ E. A. Novikov, 'Dynamics and statistics of a system of vortices,', Sov. Phys. JETP 41, 937 (1971).

${ }^{7}$ P. Tong and W. I. Goldburg, "Experimental study of relative velocity fluctuations in turbulence," Phys. Lett. A 127, 147 (1988).

${ }^{8}$ P. Tong, W. I. Goldburg, C. K. Chan, and A. Sirivat, "Turbulent transition by photon-correlation spectroscopy,', Phys. Rev. A 37, 2125 (1988).

${ }^{9} \mathrm{P}$. Tong and W. I. Goldburg, "Relative velocity fluctuations in turbulent flows at moderate Reynolds numbers. I. Experimental," Phys. Fluids 31, 2841 (1988).

${ }^{10}$ J. B. Weiss and J. C. McWilliams, "Nonergodicity of point vortices," Phys. Fluids A 3, 835 (1991).

${ }^{11}$ W. Feller, An Introduction to Probability Theory and its Applications (Wiley, New York, 1971), Vol. II.

${ }^{12}$ B. B. Mandelbrot, The Fractal Geometry of Nature (Freeman, San Francisco, 1983).

${ }^{13}$ E. W. Montroll and M. F. Shlesinger, "Maximum entropy formalism, fractals, scaling phenomena, and 1/f noise: A tale of tails," J. Stat. Phys. 32, 209 (1983).

${ }^{14} \mathrm{H}$. Takayasu, "Stable distribution and Lévy process in fractal turbulence,' Prog. Theor. Phys. 72, 471 (1984).

${ }^{15}$ M. F. Shlesinger, G. M. Zaslavsky, U. Frisch, in Lévy Flights and Related Topics in Physics, Proceedings of the International workshop held at Nice, France, June (Springer-Verlag, Berlin, 1994).

${ }^{16}$ T. S. Lundgren and Y. B. Pointin, "Statistical mechanics of twodimensional vortices," J. Stat. Phys. 17, 323 (1977).

${ }^{17} \mathrm{H}$. Aref, "Integrable, chaotic, and turbulent vortex motion in twodimensional flows," Annu. Rev. Fluid Mech. 15, 345 (1983).

${ }^{18}$ C. C. Lim, "Quasi-periodic dynamics of designularized vortex models," Physica D 37, 497 (1989).

${ }^{19} \mathrm{~J}$. C. McWilliams, "The emergence of isolated coherent vortices in turbulent flow," J. Fluid Mech. 146, 21 (1984).

${ }^{20} \mathrm{~J}$. A. Viecelli, "Statistical mechanics and correlation properties of a rotating two-dimensional flow of like-sign vortices," Phys Fluids A 5, 2484 (1993).

${ }^{21}$ A. Babiano, G. Boffeta, A. Provenzale, and A. Vulpiani, " Chaotic advection in point vortex models and two-dimensional turbulence," Phys. Fluids 6, 2465 (1994).

${ }^{22}$ A. Leonard, "Vortex methods for flow simulation," J. Comput. Phys. 37 289 (1980).

${ }^{23}$ G. S. Winckelmans and A. Leonard, "Contributions to vortex particle methods for the computation of three-dimensional incompressible unsteady flows,"' J. Comput. Phys. 109, 247 (1993).

${ }^{24}$ I. A. Ibragimov and Yu. V. Linnik, Independent and Stationary Sequences of Random Variables (Wolters-Noordhoff, Groningen, 1971).

${ }^{25}$ S. Chandrasekhar, "Stochastic problems in physics and astronomy," Rev. Mod. Phys. 15, 1 (1943).

${ }^{26} \mathrm{G}$. K. Batchelor, The Theory of Homogeneous Turbulence (Cambridge University Press, Cambridge, 1953).

${ }^{27} \mathrm{H}$. Tennekes and J. L. Lumley, A Firrst Course in Turbulence (MIT Press, Cambridge, 1972).

${ }^{28}$ F. Anselmet, Y. Gagne, and E. J. Hopfinger, "High-order velocity functions in turbulent shear flows," J. Fluid Mech. 140, 63 (1984).

${ }^{29}$ B. Castaing, Y. Gagne, and E. J. Hopfinger, "Velocity probability density functions of high Reynolds number turbulence," Physica D 46, 177 (1990).

${ }^{30} \mathrm{U}$. Frisch and Z-S. She, "On the probability density function of velocity gradients in fully developed turbulence," Fluid Dyn. Res. 8, 139 (1991).

${ }^{31}$ R. Benzi, L. Biferale, G. Paladin, A. Vulpiani, and M. Vergassola, "Multifractality in the statistics of the velocity gradients in turbulence," Phys. Rev. Lett. 67, 2299 (1991).

${ }^{32}$ R. H. Kraichnan, "Models of intermittency in hydrodynamic turbulence," Phys. Rev. Lett. 65, 575 (1990). 
${ }^{33}$ S. Kida, "Log-stable distribution and intermittency of turbulence," J. Phys. Soc. Japan, 60 (1), 5 (1991).

${ }^{34}$ A. Onuki, "Distribution of relative velocity in turbulence," Phys. Lett. A 127, 143 (1988).

${ }^{35} \mathrm{Y}$. Taguchi and H. Takayasu, "Lorentzian distribution of interacting vortex tubes," Phys. Rev. A 41, 2249 (1990).

${ }^{36}$ R. H. Kraichnan, "Intermittency in the very small scales of turbulence," Phys. Fluids 10, 2080 (1967).

${ }^{37}$ I. A. Min, "Transport, stirring and mixing in two-dimensional vortex flows," Ph.D. thesis, California Institute of Technology, 1994.
${ }^{38}$ D. I. Pullin and P. G. Saffman, "Long-time symplectic integration: The example of four-vortex motion," Proc. R. Soc. London Ser. A 432, 481 (1991).

${ }^{39}$ D. Montgomery and G. Joyce, "Statistical mechanics of 'negative temperature' states,' Phys. Fluids 17, 1139 (1974).

${ }^{40}$ R. Kraichnan and D. Montgomery, "Two-dimensional turbulence," Rep. Prog. Phys. 43, 547 (1980).

${ }^{41}$ I. Mezić, "On the statistical properties of motion of point vortices," UCSB preprint, 1995. 